\title{
Effect of Photon Energy on Conventional Intensity- Modulated Radiotherapy and Rapid Arc Radiotherapy Planning for Deep-Seated Targets in Carcinoma Cervix
}

\author{
Manindra Bhushan ${ }^{1,2}$ Girigesh Yadav ${ }^{1}$ Deepak Tripathi ${ }^{2}$ Lalit Kumar ${ }^{1,3}$ Abhinav Dewan ${ }^{1}$ \\ Inderjit Kaur Wahi ${ }^{1}$ Mahamood Suhail ${ }^{1}$ Swarupa Mitra ${ }^{1}$ Munish Gairola ${ }^{1}$
}

${ }^{1}$ Division of Medical Physics and Department of Radiation Oncology, Rajiv Gandhi Cancer Institute and Research Centre, New Delhi, India

${ }^{2}$ Amity School of Applied Sciences, Amity University (AUUP), Noida, Uttar Pradesh, India

${ }^{3}$ Dr. APJ Abdul Kalam Technical University, Lucknow, Uttar Pradesh, India

Asian J Oncol 2019;5:24-33

\begin{abstract}
Address for correspondence Manindra Bhushan, MSc, DRP, Division of Medical Physics, Department of Radiation Oncology, Rajiv Gandhi Cancer Institute and Research Centre, Sector-5, Rohini, New Delhi-110085, India (e-mail: manindra.drp44@gmail.com).
\end{abstract}

\begin{abstract}
Keywords

- intensity modulated radiation therapy

- dual arc

- non-target areas

- conformity

- homogeneity

Introduction To evaluate the dosimetric effect of photon energies on fixed field intensity-modulated radiotherapy (IMRT) and dual arc (DA) planning and to compare the dosimetric differences between conventional IMRT and DA radiotherapy planning. Materials and Methods IMRT and DA plans were generated for 15 patients having cervical cancer using different photon energies. IMRT and DA plans were generated using seven fields and double arcs, respectively. Dosimetric comparison was done in terms of planning target volume (PTV) coverage, sparing of organ at risk (OAR), homogeneity index $(\mathrm{HI})$, conformity index $(\mathrm{Cl})$, and monitor units (MUs). Photo-neutron (energy $\leq 10 \mathrm{MV}$ ) contribution was not considered for this study. Near region (NR) and far region (FR) were contoured to evaluate the dose deposited in nontarget area.

Results No significant difference was observed $(p>0.05)$ in PTV coverage for conventional IMRT and DA; however, $6 \mathrm{MV}$ yielded significantly better coverage over $15 \mathrm{MV}(p<0.05)$ for both the treatment modalities. Mean bladder dose was significantly more for conventional IMRT compared with DA. For rectal mean dose, $p$-value was nonsignificant for IMRT in comparison to DA, while significant difference was observed for change in photon energies for both treatment modalities respectively, except for $10 \mathrm{MV}$ versus $15 \mathrm{MV}$ DA plans. Significant improvements in HI (except $6 \mathrm{MV}$ vs.10 MV DA), $\mathrm{Cl}$ (except $6 \mathrm{MV}$ vs. $10 \mathrm{MV}$ IMRT and DA), MUs, NR, and FR were noted.

Conclusion DA generates more conformal, homogenous plans, requires less numbers of MUs, and deposits fewer doses to NR and FR regions of nontarget tissues in comparison to conventional IMRT. Although increase in photon energy for IMRT and DA plans reduces numbers of MUs and dose deposited to NR and FR regions, yet the choice for treatment of carcinoma cervix remains $6 \mathrm{MV}$ due to production of photo-neutrons at higher energies.
\end{abstract}

published online July 19, 2019
DOI https://doi.org/

$10.1055 / \mathrm{s}-0039-1693523$ ISSN 2454-6798.
(C2019 Spring Hope Cancer

Foundation \& Young Oncologist Group of Asia
License terms

() (1) $\ominus \circledast$ 


\section{Introduction}

Carcinoma cervix is the second most common cancer in women worldwide. The worldwide incidence of cervical cancer is approximately 510,000 new cases annually, with approximately 288,000 deaths. ${ }^{1}$ Cervical cancer is ranked as the most frequent cancer in women in India. India has a population of approximately 469.1 million women above 15 years of age, who are at risk of developing cervical cancer. The current estimates indicate approximately 96922 newly diagnosed cases and 60078 deaths annually, accounting for nearly one-third of the global cervical cancer deaths. ${ }^{2}$

Radiation therapy plays a significant role in the treatment of carcinoma of cervix. A CT- based box-technique was commonly used to deliver a conformal plan before evolution of intensity-modulated treatment. The common practice for delivering radiation by box-technique was to use high-energy photon beams. Radiotherapy (RT) has undergone a major evolution from conventional 2D planning to intensity-modulated radiation therapy (IMRT) and rapid arc planning.

IMRT is a form of radiation therapy that conforms the target volumes with prescription dose with the help of modulated intensity maps and helps in sparing of the surrounding normal tissues. ${ }^{3}$ IMRT uses different beams from different directions, mainly coplanar, to deliver nonuniform fluence maps. These beams are optimized to deliver required dose to planning target volume (PTV), leading to a better tumor control and relatively lesser dose to nearby organs at risk (OARs), resulting in reduced complications., ${ }^{4,5}$ The recent advancement in IMRT led to delivery of radiation during gantry motion with variable dose rate, gantry speed, and multileaf collimator motion. This treatment technique is referred to as "Rapid Arc." Otto et $\mathrm{al}^{6}$ gave the concept of planning and delivery of rapid arc, which is based on volumetric-modulated arc therapy (VMAT). The objective of this technique is to attain better sparing of normal tissues with improved target volume. $^{7}$

Goal of radiation therapy is to deliver maximum dose to target and minimal dose to surrounding normal tissues. It is known that higher photon energy is required to treat deeper targets. ${ }^{8,9}$ Laughlin et al recommended energy of more than $10 \mathrm{MV}$ for treatment of these deep-seated pelvic tumors, especially for larger target volumes or larger patient size, due to significant reduction in integral dose..$^{10,11}$

The aim of the present study is to compare the dosimetric effect of different photon energies on conventional fixed field IMRT and dual-rapid arc (DA) and to compare the dosimetric differences between conventional IMRT and DA radiotherapy planning in terms of PTV coverage and doses to OARs.

\section{Materials and Methods}

\section{Patient Selection and Planning Objectives}

A cohort of 15 patients with carcinoma cervix was included in the present study and referred for radiotherapy. Target volumes were almost similar for all the selected patients. The planning computed tomography (CT) scans of 3.0-mm thickness from $\mathrm{L} 2$ to proximal one-third femoral diaphysis were acquired on our CT unit (Somatom Sensation Open; Siemens, Germany).

The delineated OARs were bladder, rectum, bowel, and the femoral heads. Clinical target volume (CTV) included the cervix, uterus, parametrial tissues, and the presacral and pelvic nodes. PTV was defined by adding a 5-mm margin to CTV. Radiation therapy oncology group (RTOG) guidelines were followed for delineation of the contours. ${ }^{12}$ Plans were generated retrospectively for each patient to deliver a dose of $50.4 \mathrm{~Gy} / 28$ fractions to the PTV. To ensure uniformity of the plans, the same radiation oncologist-medical physicist duo did all the contouring and treatment planning.

\section{Planning Techniques}

For all patients, isocentric plans were made on Varian Eclipse Treatment Planning System (version 10.0) [Varian Medical Systems, Inc., Palo Alto, CA, United States] with $6 \mathrm{MV}, 10 \mathrm{MV}$, and $15 \mathrm{MV}$ photon energies. The course of treatment was scheduled for Varian TrueBeam, equipped with high definition multileaf collimator (HDMLC) with 120 leaves. Varian true beam is characterized by spatial resolution of $2.5 \mathrm{~mm}$ at isocenter for central 32 leaves and $5.0 \mathrm{~mm}$ in the outer 14 leaves on both sides, with maximum leaf speed of $4.8 \mathrm{~cm} / \mathrm{s}$ and leaf transmission of $1.4 \%$.

Fixed field sliding window conventional IMRT plans were generated with optimization of beam intensity for each gantry angle. Identical gantry angles $\left(\mathrm{G} 60^{\circ}, \mathrm{G} 100^{\circ}, \mathrm{G} 135^{\circ}, \mathrm{G} 180^{\circ}\right.$, $\mathrm{G} 225^{\circ}, \mathrm{G} 260^{\circ}$, and $\mathrm{G} 300^{\circ}$ ) with collimator $0^{\circ}$ and dose rate of $600 \mathrm{MU} / \mathrm{min}$ were used for each IMRT plan to ensure the uniformity of the study. Normal tissue objective (NTO) was used during optimization to help in sparing of normal tissues. The planning system uses direct volume optimization (DVO) to modify the intensity map to achieve the constraints given to the system. DVO controls the complexity of the plan and designs the fluence in deliverable plan. Leaf motion calculation was performed after optimization, and final calculation was done using analytic anisotropic algorithm (AAA) with grid size of $2.5 \mathrm{~mm}^{13}$

Similarly, rapid arc planning was done with the help of progressive resolution optimizer (PRO) algorithm that determines the combination of beam weights and shapes. PRO plays with dynamic variables such as gantry speed, multileaf collimator (MLC) motion, and machine dose rate and runs iterations at different levels. ${ }^{14}$ AAA was used for final dose calculation. Rapid arc plans with 6 MV, 10 MV, and 15 MV were generated for each patient with double coplanar full arcs (counter-clockwise from gantry angle $179-181^{\circ}$ with collimator angle $30^{\circ}$; clockwise from gantry angle $181-179^{\circ}$ with collimator angle $330^{\circ}$ ) with dose rate of $600 \mathrm{MU} / \mathrm{min}$.

\section{Dosimetric Variables}

Cumulative dose-volume histogram was used to evaluate the optimized plans. The various parameters compared were:

- Conformity index: $\left(\mathrm{CI}_{98}\right)$

- Homogeneity index: (HI)

- PTV: $\mathrm{V}_{93 \%}, \mathrm{~V}_{95 \%}, \mathrm{~V}_{98 \%}, \mathrm{~V}_{100 \%}, \mathrm{~V}_{105 \%}, \mathrm{~V}_{107 \%}, \mathrm{~V}_{110 \%}, \mathrm{D}_{98 \%}, \mathrm{D}_{50 \%}, \mathrm{D}_{2 \%}$, $\mathrm{D}_{\text {Min }}, \mathrm{D}_{\text {Max }}, \mathrm{D}_{\text {Mean }}, \mathrm{D}_{\text {Modal }}, \mathrm{D}_{\text {Median }}$ 
- Bladder: $\mathrm{V}_{50 G y}, \mathrm{~V}_{40 G y}, \mathrm{~V}_{30 G y}, \mathrm{~V}_{20 G y}, \mathrm{D}_{2 \%}, \mathrm{D}_{\mathrm{Max}}, \mathrm{D}_{\text {Mean }}$

- Rectu $\mathrm{V}_{50 G y}, \mathrm{~V}_{40 G y}, \mathrm{~V}_{30 G \mathrm{y}}, \mathrm{V}_{20 \mathrm{~Gy}}, \mathrm{D}_{2 \%}, \mathrm{D}_{\text {Max }}, \mathrm{D}_{\text {Mean }}$

- Bowel: $\mathrm{V}_{50 G y}, \mathrm{~V}_{40 G y}, \mathrm{~V}_{30 G \mathrm{~Gy}^{\prime}} \mathrm{V}_{20 \mathrm{~Gy}}, \mathrm{~V}_{10 \mathrm{~Gy}}, \mathrm{D}_{\text {Max }}, \mathrm{D}_{\text {Mean' }}$, $\mathrm{V}_{195 \mathrm{cc}}$ (i.e., dose received by 195 cc volume)

- Femoral heads: $\mathrm{V}_{50 G y}, \mathrm{~V}_{40 \mathrm{~Gy}}, \mathrm{~V}_{30 \mathrm{~Gy}}, \mathrm{~V}_{20 \mathrm{~Gy}}, \mathrm{~V}_{10 \mathrm{~Gy}}, \mathrm{D}_{\mathrm{Max}}, \mathrm{D}_{\text {Mean }}$

To evaluate the dose deposited near target and in regions distant from the target, nontarget tissues were divided into two regions: Near region (NR)-a shell of tissue $2.5 \mathrm{~cm}$ in radius surrounding the PTV and far region (FR)-nontarget tissue other than NR. Mean dose to NR and FR and total MUs were also compared.

For comparing the homogeneity of different plans, HI was used. The value of HI should be 1 ideally. ${ }^{15}$ It is a ratio of dose received by $2 \%$ volume of PTV to the dose received by $98 \%$ volume of the PTV, that is,

$\mathrm{HI}=\mathrm{D}_{2 \%} / \mathrm{D}_{98 \%}$

Similarly, the $\mathrm{CI}_{98}$ is given by the ratio of PTV Volume of 98\% dose coverage and the total volume of PTV. The CI having value near to 1 shows highly conformal plan. ${ }^{16}$ Hence,

$\mathrm{CI}=$ (volume of PTV covered by $98 \%$ of prescription dose) total PTV volume).

\section{Statistical Analysis}

For comparison of IMRT and dual arc (DA) plans, paired $t$-test was used. Data were considered as significant for $p \leq 0.05$. The analysis was done using the Statistical Package for Social Sciences, version 20.0 (SPSS, Chicago, Illinois, United States).

\section{Results}

\section{Target Volume Coverage}

Clinically acceptable plans were generated for conventional IMRT and DA using $6 \mathrm{MV}, 10 \mathrm{MV}$, and $15 \mathrm{MV}$ energies. The average volume of PTV in the present study was 1,479.29 \pm 242.91 $\mathrm{cm}^{3}$. No significant difference $(p>0.05)$ was observed in PTV coverage $\left(D_{98 \%}\right)$ for conventional IMRT and DA; however, change in energy (6 MV vs. 15 MV) led to significant difference in PTV coverage. Similar results were observed for $\mathrm{D}_{2 \%}(\mathrm{~Gy})$. Significant difference in $\mathrm{CI}_{98}$ was seen between both modalities for each energy $(p<0.05)$. HI results did not significantly differ between the two modalities; however, the $p$-value was significant for 6 MV over $10 \mathrm{MV}$, and $15 \mathrm{MV}$ respectively (-Table 1, - Fig. 1).

\section{OAR}

\section{Bladder}

The doses evaluated for bladder in each plan were within tolerance. Percentage volume of bladder receiving $40 \mathrm{~Gy}\left(\mathrm{~V}_{40}\right)$ and $30 \mathrm{~Gy}\left(\mathrm{~V}_{30}\right)$ decreased significantly $(p<0.05)$ with DA in comparison to IMRT ( - Table 2, - Fig. 2).

\section{Rectum}

Plans were evaluated for rectal doses for different photon energies and a significant difference was found $(p<0.05)$ for lower doses. It was observed that the volume of rectum receiving $30 \mathrm{~Gy}\left(\mathrm{~V}_{30 \mathrm{~Gy}}\right)$ was significantly less $(p<0.05)$ in case of DA when compared with IMRT (-Table 2 ).

\section{Bowel}

Evaluated doses for contoured bowel showed significant difference for lower doses such as $\mathrm{V}_{30 \mathrm{~Gy}}, \mathrm{~V}_{20 \mathrm{~Gy}}$, and $\mathrm{V}_{10 \mathrm{~Gy}}$ with DA plan being better than IMRT plan. Similarly, the dose received by $195 \mathrm{~mL}$ of bowel was significantly lesser in case of DA (-Table 2).

\section{Femoral Heads}

Mean dose to femur was higher for IMRT than DA plans for their respective energies (- Table 2 ) ( - Fig. 3 ).

\section{Near Region and Far Region}

To evaluate the peripheral dose, the contoured body tissues were divided into two structures: NR, a shell of radius $2.5 \mathrm{~cm}$ around PTV and FR, a region in nontarget normal tissue area outside the near region. ${ }^{17}$ The mean doses received by NR and FR remained similar in both the modalities $(p>0.05)$ for each energy (-Table 2).

\section{Total Monitor Units and Beam-On Time}

Total monitor units (TMUs) resulting in optimized plans were significantly less for DA plans. Monitor units were 3.12 times more for IMRT $_{6 \mathrm{MV}}$ versus $\mathrm{DA}_{6 \mathrm{MV}}, 3.21$ times more for IMRT $\mathrm{IMMV}_{10}$ versus $\mathrm{DA}_{10 \mathrm{Mv}}$ and 3.27 times more for IMRT $\mathrm{IMMv}_{15 \mathrm{Mv}}$ versus DA $(p<0.05)$ ( - Table 2). BOT differed between two modalities ( $p$ $<0.05$ ) for each photon energy as the time required to deliver planned TMU was lesser for DA, considering the fact that patient setup and image verification time was same for all patients.

\section{Discussion}

IMRT plans use different static gantry angles to provide deliverable intensity maps, which may result in delivery of excessive dose during the transmission through the body tissues as entry doses. ${ }^{11,18}$ It can lead to increase in integral dose as well as the increased risk of secondary malignancies. ${ }^{19,20} \mathrm{At}$ the same time, VMAT uses continuous rotation of gantry to deliver similar or even better optimized plan due to increase in the degree of freedom.

Various studies using DA technique in head and neck cancers have concluded ${ }^{21,22}$ that an equivalent target volume coverage and normal tissue constraints can be achieved by VMAT. Cozzi et al studied the nature of plans by fixed-field IMRT and volumetric arc therapy in gynecological malignancies with $6 \mathrm{MV}$ photon energy ${ }^{23}$ and found similar results. The impact of target volume over OARs was also studied by the investigators. It was observed in our study that the DA had an edge over IMRT for covering target volume. Law et $\mathrm{al}^{24}$ and Ost et $\mathrm{al}^{25}$ have published similar studies for prostate carcinoma, whereas Blomquist et al have compared different energies for treatment of lung cancers. ${ }^{26}$

As per The International Commission on Radiation Units and Measurements 83 (ICRU 83) guidelines, the dose to $98 \%$ target volume was taken into account $\left(D_{98 \%}\right)$ for PTV coverage. It was observed that $6 \mathrm{MV}$ photon energy was superior to $15 \mathrm{MV}$ photon energy even with change in treatment technique, but showed no variation when 
Table 1 DVH analysis of planning target volume for IMRT and DA for 6 MV, $10 \mathrm{MV}$, and $15 \mathrm{MV}$ and their respective $p$-value

\begin{tabular}{|c|c|c|c|c|c|c|c|c|c|c|c|c|c|c|c|}
\hline \multirow{3}{*}{\begin{tabular}{|l|} 
PTV \\
Parameter \\
\end{tabular}} & \multicolumn{9}{|c|}{ Energy } & \multicolumn{6}{|c|}{$p$-Value } \\
\hline & \multicolumn{3}{|c|}{$6 \mathrm{MV}$} & \multicolumn{3}{|c|}{$10 \mathrm{MV}$} & \multicolumn{3}{|c|}{$15 \mathrm{MV}$} & \multicolumn{3}{|c|}{ IMRT } & \multicolumn{3}{|c|}{ DA } \\
\hline & IMRT & $\mathrm{DA}$ & $p$-Value & IMRT & DA & $p$-Value & IMRT & DA & $p$-Value & $\begin{array}{l}6 \text { vs. } \\
10\end{array}$ & $\begin{array}{l}10 \text { vs. } \\
15\end{array}$ & $\begin{array}{l}6 \text { vs. } \\
15\end{array}$ & $\begin{array}{l}6 \text { vs. } \\
10\end{array}$ & $\begin{array}{l}10 \text { vs. } \\
15\end{array}$ & $\begin{array}{l}6 \text { vs. } \\
15\end{array}$ \\
\hline $\mathrm{V}_{95}(\%)$ & $\begin{array}{l}99.91 \pm \\
0.36\end{array}$ & $\begin{array}{l}100.0 \pm \\
0.00\end{array}$ & NS & $\begin{array}{l}99.90 \pm \\
0.36\end{array}$ & $\begin{array}{l}100.0 \pm \\
0.00\end{array}$ & NS & $\begin{array}{l}99.91 \pm \\
0.36\end{array}$ & $\begin{array}{l}100.0 \pm \\
0.00\end{array}$ & NS & NS & NS & NS & NS & NS & NS \\
\hline $\mathrm{V}_{98}(\%)$ & $\begin{array}{l}97.90 \pm \\
0.71\end{array}$ & $\begin{array}{l}97.48 \pm \\
1.02\end{array}$ & NS & $\begin{array}{l}98.01 \pm \\
0.70\end{array}$ & $\begin{array}{l}97.97 \pm \\
0.91\end{array}$ & NS & $\begin{array}{l}98.16 \pm \\
0.75\end{array}$ & $\begin{array}{l}98.30 \pm \\
0.67\end{array}$ & NS & NS & NS & 0.021 & NS & NS & 0 \\
\hline $\mathrm{V}_{100}(\%)$ & $\begin{array}{l}94.05 \pm \\
1.95\end{array}$ & $\begin{array}{l}93.07 \pm \\
2.38\end{array}$ & NS & $\begin{array}{l}93.97 \pm \\
2.11\end{array}$ & $\begin{array}{l}94.02 \pm \\
1.77\end{array}$ & NS & $\begin{array}{l}94.21 \pm \\
2.14\end{array}$ & $\begin{array}{l}94.68 \pm \\
1.30\end{array}$ & NS & NS & NS & 0.047 & NS & NS & 0.002 \\
\hline $\mathrm{V}_{110}(\%)$ & $\begin{array}{l}1.85 \pm \\
1.56\end{array}$ & $\begin{array}{l}1.31 \pm \\
1.59\end{array}$ & NS & $\begin{array}{l}1.40 \pm \\
1.62\end{array}$ & $\begin{array}{l}0.70 \pm \\
1.11\end{array}$ & NS & $\begin{array}{l}0.97 \pm \\
1.09\end{array}$ & $\begin{array}{l}1.37 \pm \\
2.30\end{array}$ & NS & 0.048 & NS & 0 & 0.03 & NS & 0.046 \\
\hline$D_{98 \%}(G y)$ & $\begin{array}{l}49.35 \pm \\
0.43\end{array}$ & $\begin{array}{l}49.20 \pm \\
0.39\end{array}$ & NS & $\begin{array}{l}49.37 \pm \\
0.41\end{array}$ & $\begin{array}{l}49.41 \pm \\
0.43\end{array}$ & NS & $\begin{array}{l}49.45 \pm \\
0.43\end{array}$ & $\begin{array}{l}49.52 \pm \\
0.31\end{array}$ & NS & NS & 0.023 & 0.023 & 0.034 & NS & 0 \\
\hline$D_{50 \%}(G y)$ & $\begin{array}{l}52.39 \pm \\
0.45\end{array}$ & $\begin{array}{l}52.77 \pm \\
0.52\end{array}$ & 0.018 & $\begin{array}{l}52.33 \pm \\
0.51\end{array}$ & $\begin{array}{l}52.89 \pm \\
0.41\end{array}$ & 0.003 & $\begin{array}{l}52.28 \pm \\
0.46\end{array}$ & $\begin{array}{l}52.93 \pm \\
0.46\end{array}$ & 0.001 & NS & NS & 0 & NS & NS & 0.046 \\
\hline $\mathrm{D}_{2 \%}$ (Gy) & $\begin{array}{l}55.24 \pm \\
0.54\end{array}$ & $\begin{array}{l}54.93 \pm \\
0.71\end{array}$ & NS & $\begin{array}{l}54.93 \pm \\
0.58\end{array}$ & $\begin{array}{l}54.84 \pm \\
0.49\end{array}$ & NS & $\begin{array}{l}54.78 \pm \\
0.53\end{array}$ & $\begin{array}{l}54.97 \pm \\
0.68\end{array}$ & NS & 0.002 & 0.033 & 0 & NS & NS & NS \\
\hline $\mathrm{D}_{\min }(\mathrm{Gy})$ & $\begin{array}{l}42.01 \pm \\
2.86\end{array}$ & $\begin{array}{l}43.39 \pm \\
2.50\end{array}$ & NS & $\begin{array}{l}42.45 \pm \\
2.58\end{array}$ & $\begin{array}{l}43.66 \pm \\
2.35\end{array}$ & NS & $\begin{array}{l}43.20 \pm \\
2.59\end{array}$ & $\begin{array}{l}44.27 \pm \\
2.35\end{array}$ & NS & NS & 0.026 & 0.004 & 0.033 & 0.002 & 0 \\
\hline$D_{\max }(G y)$ & $\begin{array}{l}58.89 \pm \\
1.31\end{array}$ & $\begin{array}{l}57.56 \pm \\
1.11\end{array}$ & 0.005 & $\begin{array}{l}58.11 \pm \\
1.31\end{array}$ & $\begin{array}{l}56.81 \pm \\
0.84\end{array}$ & 0.002 & $\begin{array}{l}57.76 \pm \\
1.03\end{array}$ & $\begin{array}{l}57.35 \pm \\
1.07\end{array}$ & NS & 0 & 0.014 & 0 & 0 & 0 & 0.034 \\
\hline$D_{\text {mean }}(G y)$ & $\begin{array}{l}52.39 \pm \\
0.40\end{array}$ & \begin{tabular}{|l|}
$52.62 \pm$ \\
0.48
\end{tabular} & NS & $\begin{array}{l}52.31 \pm \\
0.47\end{array}$ & $\begin{array}{l}52.71 \pm \\
0.36\end{array}$ & NS & $\begin{array}{l}52.26 \pm \\
0.42\end{array}$ & $\begin{array}{l}52.78 \pm \\
0.43\end{array}$ & 0.003 & NS & NS & 0 & NS & NS & 0.04 \\
\hline $\mathrm{D}_{\text {modal }}(G y)$ & $\begin{array}{l}52.36 \pm \\
0.50\end{array}$ & $\begin{array}{l}52.93 \pm \\
0.55\end{array}$ & 0.001 & $\begin{array}{l}52.29 \pm \\
0.62\end{array}$ & $\begin{array}{l}53.08 \pm \\
0.46\end{array}$ & 0.001 & $\begin{array}{l}52.20 \pm \\
0.51\end{array}$ & $\begin{array}{l}53.08 \pm \\
0.49\end{array}$ & 0 & NS & NS & 0.008 & NS & NS & NS \\
\hline $\mathrm{D}_{\text {median }}(\mathrm{Gy})$ & $\begin{array}{l}52.40 \pm \\
0.45\end{array}$ & \begin{tabular}{|l|}
$52.77 \pm$ \\
0.51
\end{tabular} & 0.017 & $\begin{array}{l}52.33 \pm \\
0.52\end{array}$ & $\begin{array}{l}52.89 \pm \\
0.40\end{array}$ & 0.003 & $\begin{array}{l}52.28 \pm \\
0.46\end{array}$ & $\begin{array}{l}52.943 \pm \\
0.46\end{array}$ & 0.001 & NS & NS & 0 & NS & NS & 0.044 \\
\hline GM & $\begin{array}{l}4.36 \pm \\
0.415\end{array}$ & $\begin{array}{l}3.971 \pm \\
0.317\end{array}$ & 0 & $\begin{array}{l}3.962 \pm \\
0.291\end{array}$ & $\begin{array}{l}3.774 \pm \\
0.278\end{array}$ & 0.001 & $\begin{array}{l}3.808 \pm \\
0.270\end{array}$ & $\begin{array}{l}3.692 \pm \\
0.275\end{array}$ & 0.025 & 0 & 0 & 0 & 0 & 0 & 0 \\
\hline $\mathrm{HI}$ & $\begin{array}{l}1.119 \pm \\
0.015\end{array}$ & $\begin{array}{l}1.114 \pm \\
0.017\end{array}$ & NS & $\begin{array}{l}1.113 \pm \\
0.013\end{array}$ & $\begin{array}{l}1.111 \pm \\
0.014\end{array}$ & NS & $\begin{array}{l}1.107 \pm \\
0.013\end{array}$ & $\begin{array}{l}1.107 \pm \\
0.015\end{array}$ & NS & 0.003 & 0.006 & 0 & 0.06 & NS & 0.067 \\
\hline $\mathrm{Cl}$ & $\begin{array}{l}1.144 \pm \\
0.116\end{array}$ & $\begin{array}{l}1.094 \pm \\
0.089\end{array}$ & 0 & $\begin{array}{l}1.125 \pm \\
0.112\end{array}$ & $\begin{array}{l}1.086 \pm \\
0.082\end{array}$ & 0.003 & $\begin{array}{l}1.122 \pm \\
0.113\end{array}$ & $\begin{array}{l}1.005 \pm \\
.0870\end{array}$ & 0.004 & 0 & NS & 0 & 0.004 & NS & 0 \\
\hline
\end{tabular}

Abbreviations: $\mathrm{Cl}$, conformity index; DA, dual arc; Dmax, maximum dose; Dmean, mean dose; Dmedian, median dose; Dmin, minimum dose; Dmodal, modal dose; GM, gradient measure; $\mathrm{HI}$, homogeneity index; IMRT, intensity modulated radiotherapy; NS, nonsignificant; PTV, planning target volume; $\mathrm{V}_{\mathrm{x}}(\%)$, $\%$ of PTV receiving $\mathrm{X} \%$ of prescription dose.

photon energy remained same. ${ }^{8,17}$ This can be explained by lower dose contribution of lower photon energy to the dose coverage. The other parameters used were $\mathrm{CI}_{98}$ and $\mathrm{HI}$. The results shown were in favor of $6 \mathrm{MV}$ due to the same reason.

It was clear that DA was superior to IMRT for bladder doses. Hence, the calculated $p$-value for mean bladder dose was significantly more for conventional IMRT in comparison to DA $(p<0.05)$ for change in photon energies for both treatment modalities, except for $6 \mathrm{MV}$ versus $10 \mathrm{MV}(p>0.05)$. For rectal mean dose, $p$-value was not significant $(p>0.05)$ for IMRT in comparison to DA, but significantly differed $(p<0.05)$ with change in photon energies for both treatment modalities, except $10 \mathrm{MV}$ versus 15 MV DA plans.

The rapid arc technique includes all the possible gantry angles, which may be missing from IMRT technique and hence increases the homogeneity of dose painting inside the target volume. Due to conformality of doses to the target volume, the mean doses to the nearby normal structures were also reduced. The mean doses of all the critical structures remain lesser in dual arc with $6 \mathrm{MV}$ photon energy, when compared with energy $\mathrm{E} \geq 10 \mathrm{MV}$.
There was no significant difference between $6 \mathrm{MV}, 10 \mathrm{MV}$, and $15 \mathrm{MV}$ photon energies for each single treatment modality for PTV coverage. These results are in accordance with the study done by Liu et al who did not observe any noticeable difference between $6 \mathrm{MV}$ and $18 \mathrm{MV}$ IMRT plans. ${ }^{27} 6 \mathrm{MV}$ plans were showing slightly better dose conformity and homogeneity. There was no significant impact of PTV location or PTV volume for the photon energy leading to superior plan quality. ${ }^{28-30}$

Mean dose of femoral heads reduced significantly in dual arcs. It may be explained on the basis of reduced scatter dose and lesser beam-on time with rapid arc technique. It was found that the total number of monitor unit in each optimized plan was significantly lesser for arc technique in comparison to IMRT, which may be explained on the basis of degree of freedom, attained by gantry angles.

Modern radiotherapy techniques have resulted in an improved survival in patients. This improvement in survival, however, increases the risk of observing a relatively rare but potentially lethal effect, that is, secondary malignancy. IMRT leads to better conformity of plans, which has resulted in improved outcome along with reduction in late normal tissue complications. 

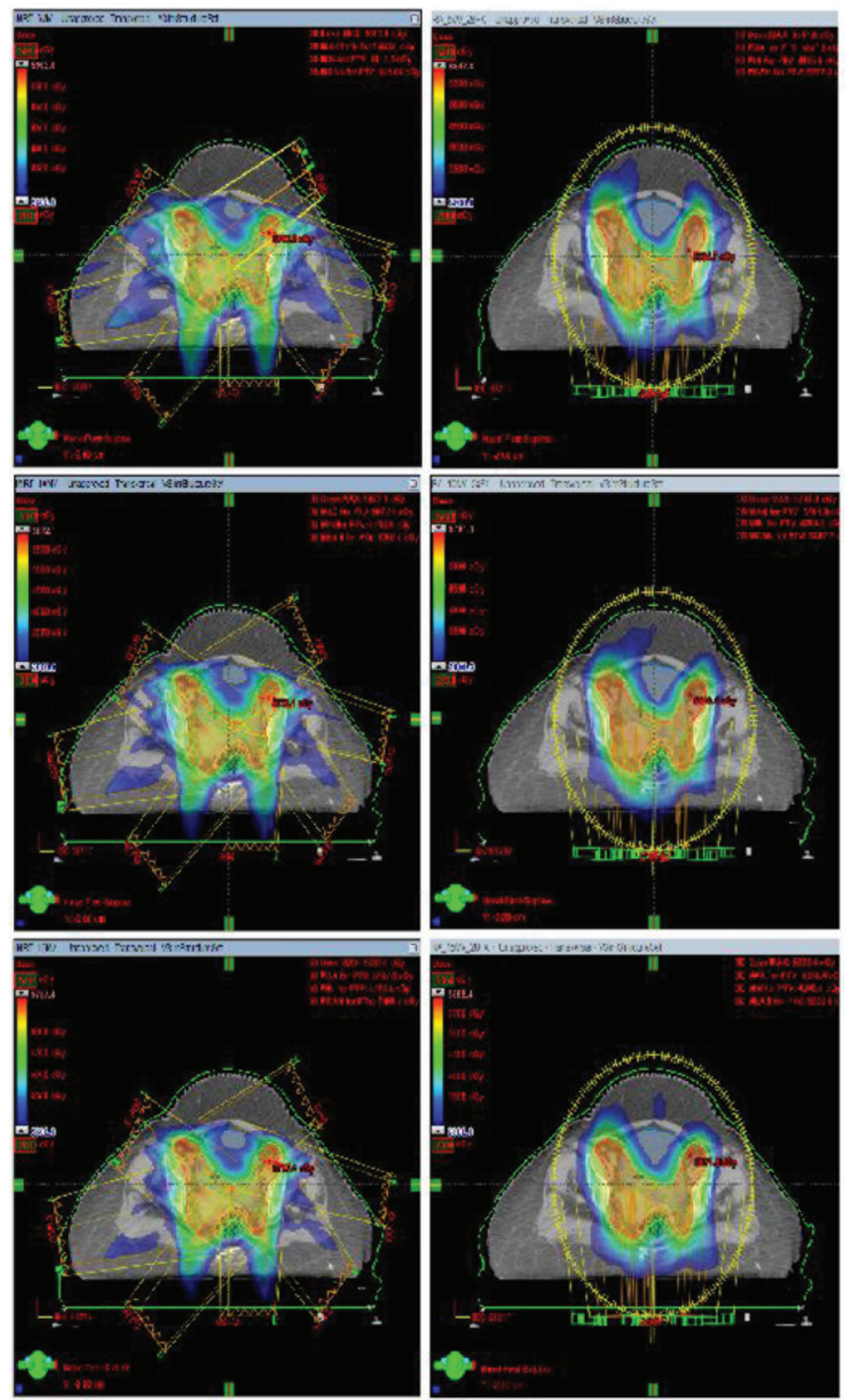

Fig. 1 Dose coverage of 95\% prescription dose for $6 \mathrm{MV}, 10 \mathrm{MV}$, and $15 \mathrm{MV}$ for IMRT and dual arc (DA). IMRT, intensity-modulated radiotherapy.

Higher conformity is however, obtained by increasing number of fields and using fixed-shape or dynamic conformal arc beams. Furthermore, increased monitor unit, difference in dose distribution, and scatter along with use of multiple coplanar or noncoplanar beams significantly increases the volume of tissue exposed to low doses of radiation. These factors have key implications over the possible increase of secondary cancers due to radiotherapy. Delivery of specified dose from IMRT/VMAT technique requires linear accelerator to be energized for longer time (more monitor units needed) and, thereby resulting in increased total body dose due to leakage radiation.

IMRT leads to increase in integral dose exposure to nontumor tissues as larger volumes of normal tissues are exposed to lower doses. This increase in energy deposition in healthy tissue leads to generation of secondary cancers.

There is a significant benefit of shorter beam-on time, observed for dual arcs for all the photon energies as compared 
Table 2 DVH analysis of OARs for IMRT and DA for $6 \mathrm{MV}, 10 \mathrm{MV}$, and $15 \mathrm{MV}$ and their respective $p$-values

\begin{tabular}{|c|c|c|c|c|c|c|c|c|c|c|c|c|c|c|c|}
\hline \multirow{3}{*}{\begin{tabular}{|l|} 
PTV \\
Parameter \\
\end{tabular}} & \multicolumn{9}{|c|}{ Energy } & \multicolumn{6}{|c|}{$p$-Value } \\
\hline & \multicolumn{3}{|c|}{$6 \mathrm{MV}$} & \multicolumn{3}{|c|}{$10 \mathrm{MV}$} & \multicolumn{3}{|c|}{$15 \mathrm{MV}$} & \multicolumn{3}{|c|}{ IMRT } & \multicolumn{3}{|c|}{ DA } \\
\hline & IMRT & DA & $p$-Value & IMRT & DA & $p$-Value & IMRT & DA & $p$-Value & $\begin{array}{l}6 \text { vs. } \\
10\end{array}$ & $\begin{array}{l}10 \text { vs. } \\
15\end{array}$ & 6 vs. 15 & $\begin{array}{l}6 \text { vs. } \\
10\end{array}$ & $\begin{array}{l}10 \text { vs. } \\
15\end{array}$ & \begin{tabular}{|l|}
6 vs. \\
15
\end{tabular} \\
\hline $\mathrm{V}_{95}(\%)$ & $\begin{array}{l}99.91 \pm \\
0.36\end{array}$ & $\begin{array}{l}100.0 \\
\pm 0.00\end{array}$ & NS & $\begin{array}{l}99.90 \pm \\
0.36\end{array}$ & $\begin{array}{l}100.0 \pm \\
0.00\end{array}$ & NS & $\begin{array}{l}99.91 \pm \\
0.36\end{array}$ & $\begin{array}{l}100.0 \pm \\
0.00\end{array}$ & NS & NS & NS & NS & NS & NS & NS \\
\hline $\mathrm{V}_{98}(\%)$ & $\begin{array}{l}97.90 \pm \\
0.71\end{array}$ & $\begin{array}{l}97.48 \\
\pm 1.02\end{array}$ & NS & $\begin{array}{l}98.01 \pm \\
0.70\end{array}$ & $\begin{array}{l}97.97 \pm \\
0.91\end{array}$ & NS & $\begin{array}{l}98.16 \pm \\
0.75\end{array}$ & $\begin{array}{l}98.30 \pm \\
0.67\end{array}$ & NS & NS & NS & 0.021 & NS & NS & 0 \\
\hline$V_{100}(\%)$ & $\begin{array}{l}94.05 \pm \\
1.95\end{array}$ & $\begin{array}{l}93.07 \\
\pm 2.38\end{array}$ & NS & $\begin{array}{l}93.97 \pm \\
2.11\end{array}$ & $\begin{array}{l}94.02 \pm \\
1.77\end{array}$ & NS & $\begin{array}{l}94.21 \pm \\
2.14\end{array}$ & $\begin{array}{l}94.68 \pm \\
1.30\end{array}$ & NS & NS & NS & 0.047 & NS & NS & 0.002 \\
\hline$V_{110}(\%)$ & $\begin{array}{l}1.85 \pm \\
1.56\end{array}$ & $\begin{array}{l}1.31 \pm \\
1.59\end{array}$ & NS & $\begin{array}{l}1.40 \pm \\
1.62\end{array}$ & $\begin{array}{l}0.70 \pm \\
1.11\end{array}$ & NS & $\begin{array}{l}0.97 \pm \\
1.09\end{array}$ & $\begin{array}{l}1.37 \pm \\
2.30\end{array}$ & NS & 0.048 & NS & 0 & 0.03 & NS & 0.046 \\
\hline $\mathrm{D}_{98 \%}(\mathrm{~Gy})$ & $\begin{array}{l}49.35 \pm \\
0.43\end{array}$ & $\begin{array}{l}49.20 \\
\pm 0.39\end{array}$ & NS & $\begin{array}{l}49.37 \pm \\
0.41\end{array}$ & $\begin{array}{l}49.41 \pm \\
0.43\end{array}$ & NS & $\begin{array}{l}49.45 \pm \\
0.43\end{array}$ & $\begin{array}{l}49.52 \pm \\
0.31\end{array}$ & NS & NS & 0.023 & 0.023 & 0.034 & NS & 0 \\
\hline$D_{50 \%}(G y)$ & $\begin{array}{l}52.39 \pm \\
0.45\end{array}$ & $\begin{array}{l}52.77 \\
\pm 0.52\end{array}$ & 0.018 & $\begin{array}{l}52.33 \pm \\
0.51\end{array}$ & $\begin{array}{l}52.89 \pm \\
0.41\end{array}$ & 0.003 & $\begin{array}{l}52.28 \pm \\
0.46\end{array}$ & $\begin{array}{l}52.93 \pm \\
0.46\end{array}$ & 0.001 & NS & NS & 0 & NS & NS & 0.046 \\
\hline$D_{2 \%}(G y)$ & $\begin{array}{l}55.24 \pm \\
0.54\end{array}$ & $\begin{array}{l}54.93 \\
\pm 0.71\end{array}$ & NS & $\begin{array}{l}54.93 \pm \\
0.58\end{array}$ & $\begin{array}{l}54.84 \pm \\
0.49\end{array}$ & NS & $\begin{array}{l}54.78 \pm \\
0.53\end{array}$ & $\begin{array}{l}54.97 \pm \\
0.68\end{array}$ & NS & 0.002 & 0.033 & 0 & NS & NS & NS \\
\hline$D_{\min }(G y)$ & $\begin{array}{l}42.01 \pm \\
2.86\end{array}$ & $\begin{array}{l}43.39 \\
\pm 2.50\end{array}$ & NS & $\begin{array}{l}42.45 \pm \\
2.58\end{array}$ & $\begin{array}{l}43.66 \pm \\
2.35\end{array}$ & NS & $\begin{array}{l}43.20 \pm \\
2.59\end{array}$ & $\begin{array}{l}44.27 \pm \\
2.35\end{array}$ & NS & NS & 0.026 & 0.004 & 0.033 & 0.002 & 0 \\
\hline$D_{\max }(G y)$ & $\begin{array}{l}58.89 \pm \\
1.31\end{array}$ & $\begin{array}{l}57.56 \\
\pm 1.11\end{array}$ & 0.005 & $\begin{array}{l}58.11 \pm \\
1.31\end{array}$ & $\begin{array}{l}56.81 \pm \\
0.84\end{array}$ & 0.002 & $\begin{array}{l}57.76 \pm \\
1.03\end{array}$ & $\begin{array}{l}57.35 \pm \\
1.07\end{array}$ & NS & 0 & 0.014 & 0 & 0 & 0 & 0.034 \\
\hline$D_{\text {mean }}(G y)$ & $\begin{array}{l}52.39 \pm \\
0.40\end{array}$ & $\begin{array}{l}52.62 \\
\pm 0.48\end{array}$ & NS & $\begin{array}{l}52.31 \pm \\
0.47\end{array}$ & $\begin{array}{l}52.71 \pm \\
0.36\end{array}$ & NS & $\begin{array}{l}52.26 \pm \\
0.42\end{array}$ & $\begin{array}{l}52.78 \pm \\
0.43\end{array}$ & 0.003 & NS & NS & 0 & NS & NS & 0.04 \\
\hline $\mathrm{D}_{\text {modal }}(G y)$ & $\begin{array}{l}52.36 \pm \\
0.50\end{array}$ & $\begin{array}{l}52.93 \\
\pm 0.55\end{array}$ & 0.001 & $\begin{array}{l}52.29 \pm \\
0.62\end{array}$ & $\begin{array}{l}53.08 \pm \\
0.46\end{array}$ & 0.001 & $\begin{array}{l}52.20 \pm \\
0.51\end{array}$ & $\begin{array}{l}53.08 \pm \\
0.49\end{array}$ & 0 & NS & NS & 0.008 & NS & NS & NS \\
\hline$D_{\text {median }}(G y)$ & $\begin{array}{l}52.40 \pm \\
0.45\end{array}$ & $\begin{array}{l}52.77 \\
\pm 0.51\end{array}$ & 0.017 & $\begin{array}{l}52.33 \pm \\
0.52\end{array}$ & $\begin{array}{l}52.89 \pm \\
0.40\end{array}$ & 0.003 & $\begin{array}{l}52.28 \pm \\
0.46\end{array}$ & $\begin{array}{l}52.943 \pm \\
0.46\end{array}$ & 0.001 & NS & NS & 0 & NS & NS & 0.044 \\
\hline GM & $\begin{array}{l}4.36 \pm \\
0.415\end{array}$ & $\begin{array}{l}3.971 \\
\pm \\
0.317\end{array}$ & 0 & $\begin{array}{l}3.962 \pm \\
0.291\end{array}$ & $\begin{array}{l}3.774 \pm \\
0.278\end{array}$ & 0.001 & $\begin{array}{l}3.808 \pm \\
0.270\end{array}$ & $\begin{array}{l}3.692 \pm \\
0.275\end{array}$ & 0.025 & 0 & 0 & 0 & 0 & 0 & 0 \\
\hline $\mathrm{HI}$ & $\begin{array}{l}1.119 \pm \\
0.015\end{array}$ & $\begin{array}{l}1.114 \\
\pm \\
0.017\end{array}$ & NS & $\begin{array}{l}1.113 \pm \\
0.013\end{array}$ & $\begin{array}{l}1.111 \pm \\
0.014\end{array}$ & NS & $\begin{array}{l}1.107 \pm \\
0.013\end{array}$ & $\begin{array}{l}1.107 \pm \\
0.015\end{array}$ & NS & 0.003 & 0.006 & 0 & 0.06 & NS & 0.067 \\
\hline $\mathrm{Cl}$ & $\begin{array}{l}1.144 \pm \\
0.116\end{array}$ & $\begin{array}{l}1.094 \\
\pm \\
0.089\end{array}$ & 0 & $\begin{array}{l}0.125 \pm \\
0.112\end{array}$ & $\begin{array}{l}1.086 \pm \\
0.082\end{array}$ & 0.003 & $\begin{array}{l}1.122 \pm \\
0.113\end{array}$ & $\begin{array}{l}1.005 \pm \\
.0870\end{array}$ & 0.004 & 0 & NS & 0 & 0.004 & NS & 0 \\
\hline \multicolumn{16}{|l|}{ Bladder } \\
\hline $\mathrm{V}_{50}(\%)$ & $\begin{array}{l}33.01 \pm \\
5.71\end{array}$ & $\begin{array}{l}32.17 \\
\pm 6.12\end{array}$ & NS & $\begin{array}{l}32.32 \pm \\
6.02\end{array}$ & $\begin{array}{l}33.35 \pm \\
4.54\end{array}$ & NS & $\begin{array}{l}31.31 \pm \\
5.61\end{array}$ & $\begin{array}{l}32.44 \pm \\
5.10\end{array}$ & NS & 0.019 & 0.002 & 0 & NS & 0.032 & NS \\
\hline $\mathrm{V}_{40}(\%)$ & $\begin{array}{l}58.01 \pm \\
8.55\end{array}$ & $\begin{array}{l}54.60 \\
\pm 7.54\end{array}$ & 0.005 & $\begin{array}{l}57.48 \pm \\
8.38\end{array}$ & $\begin{array}{l}54.31 \pm \\
7.41\end{array}$ & 0.003 & $\begin{array}{l}56.97 \pm \\
8.54\end{array}$ & $\begin{array}{l}53.84 \pm \\
7.55\end{array}$ & 0.001 & NS & 0.002 & 0.005 & NS & 0.005 & 0.003 \\
\hline$V_{30}(\%)$ & $\begin{array}{l}84.76 \pm \\
6.34\end{array}$ & $\begin{array}{l}75.72 \\
\pm 9.82\end{array}$ & 0.001 & $\begin{array}{l}83.53 \pm \\
5.60\end{array}$ & $\begin{array}{l}74.91 \pm \\
9.70\end{array}$ & 0.002 & $\begin{array}{l}82.05 \pm \\
6.65\end{array}$ & $\begin{array}{l}73.60 \pm \\
9.90\end{array}$ & 0.001 & 0.008 & 0 & 0 & NS & 0.012 & 0.001 \\
\hline$V_{20}(\%)$ & $\begin{array}{l}98.01 \pm \\
3.10\end{array}$ & $\begin{array}{l}98.57 \\
\pm 2.39\end{array}$ & NS & $\begin{array}{l}98.01 \pm \\
3.10\end{array}$ & $\begin{array}{l}98.68 \pm \\
2.90\end{array}$ & NS & $\begin{array}{l}97.98 \pm \\
3.20\end{array}$ & $\begin{array}{l}98.30 \pm \\
3.07\end{array}$ & NS & NS & NS & NS & NS & NS & NS \\
\hline$D_{2 \%}(G y)$ & $\begin{array}{l}54.10 \pm \\
0.73\end{array}$ & $\begin{array}{l}53.93 \\
\pm 0.86\end{array}$ & NS & $\begin{array}{l}53.92 \pm \\
0.83\end{array}$ & $\begin{array}{l}53.79 \pm \\
0.68\end{array}$ & NS & $\begin{array}{l}53.62 \pm \\
0.71\end{array}$ & $\begin{array}{l}53.75 \pm \\
0.83\end{array}$ & NS & NS & 0.003 & 0 & NS & NS & 0.048 \\
\hline $\mathrm{D}_{\max }(\mathrm{Gy})$ & $\begin{array}{l}56.88 \pm \\
1.22\end{array}$ & $\begin{array}{l}56.07 \\
\pm 1.16\end{array}$ & NS & $\begin{array}{l}56.43 \pm \\
1.18\end{array}$ & $\begin{array}{l}55.71 \pm \\
0.74\end{array}$ & NS & $\begin{array}{l}55.94 \pm \\
1.12\end{array}$ & $\begin{array}{l}55.83 \pm \\
1.11\end{array}$ & NS & 0.004 & 0.001 & 0 & NS & NS & 0.25 \\
\hline $\mathrm{D}_{\text {mean }}(\mathrm{Gy})$ & $\begin{array}{l}41.90 \pm \\
1.65\end{array}$ & $\begin{array}{l}40.46 \\
\pm 2.06\end{array}$ & 0.003 & $\begin{array}{l}41.77 \pm \\
1.53\end{array}$ & $\begin{array}{l}40.31 \pm \\
1.96\end{array}$ & 0.001 & $\begin{array}{l}41.46 \pm \\
1.77\end{array}$ & $\begin{array}{l}40.01 \pm \\
2.04\end{array}$ & 0.001 & NS & 0 & 0 & NS & 0.003 & 0.01 \\
\hline \multicolumn{16}{|l|}{ Rectum } \\
\hline$V_{50}(\%)$ & $\begin{array}{l}22.70 \pm \\
8.72\end{array}$ & $\begin{array}{l}21.80 \\
\pm 7.17\end{array}$ & NS & $\begin{array}{l}24.02 \pm \\
9.05\end{array}$ & $\begin{array}{l}24.43 \pm \\
7.16\end{array}$ & NS & $\begin{array}{l}26.80 \pm \\
9.90\end{array}$ & $\begin{array}{l}25.69 \pm \\
8.10\end{array}$ & NS & 0.015 & 0.001 & 0 & 0.026 & NS & 0 \\
\hline $\mathrm{V}_{40}(\%)$ & $\begin{array}{l}75.98 \pm \\
10.02\end{array}$ & $\begin{array}{l}72.68 \\
\pm \\
13.89\end{array}$ & NS & $\begin{array}{l}77.10 \pm \\
10.68\end{array}$ & $\begin{array}{l}73.7 \pm \\
14.01\end{array}$ & NS & $\begin{array}{l}77.21 \pm \\
10.75\end{array}$ & $\begin{array}{l}74.77 \pm \\
13.66\end{array}$ & NS & 0.034 & 0.012 & 0 & NS & NS & 0.03 \\
\hline
\end{tabular}


30 Effect of Photon Energy in Carcinoma Cervix Bhushan et al.

Table 2 (continued)

\begin{tabular}{|c|c|c|c|c|c|c|c|c|c|c|c|c|c|c|c|}
\hline \multirow{3}{*}{$\begin{array}{l}\text { PTV } \\
\text { Parameter }\end{array}$} & \multicolumn{9}{|c|}{ Energy } & \multicolumn{6}{|c|}{$p$-Value } \\
\hline & \multicolumn{3}{|c|}{$6 \mathrm{MV}$} & \multicolumn{3}{|c|}{$10 \mathrm{MV}$} & \multicolumn{3}{|c|}{$15 \mathrm{MV}$} & \multicolumn{3}{|c|}{ IMRT } & \multicolumn{3}{|c|}{ DA } \\
\hline & IMRT & $\mathrm{DA}$ & $p$-Value & IMRT & DA & $p$-Value & IMRT & DA & $p$-Value & $\begin{array}{l}6 \text { vs. } \\
10\end{array}$ & $\begin{array}{l}10 \text { vs. } \\
15\end{array}$ & 6 vs. 15 & $\begin{array}{l}6 \text { vs. } \\
10\end{array}$ & $\begin{array}{l}10 \text { vs. } \\
15\end{array}$ & $\begin{array}{l}6 \text { vs. } \\
15\end{array}$ \\
\hline $\mathrm{V}_{30}(\%)$ & $\begin{array}{l}93.14 \pm \\
4.84\end{array}$ & $\begin{array}{l}89.37 \\
\pm 8.79 \\
\end{array}$ & 0.021 & $\begin{array}{l}93.13 \pm \\
5.02 \\
\end{array}$ & $\begin{array}{l}89.58 \pm \\
8.74\end{array}$ & 0.02 & $\begin{array}{l}93.12 \pm \\
5.14 \\
\end{array}$ & $\begin{array}{l}89.86 \pm \\
8.47 \\
\end{array}$ & 0.027 & NS & NS & NS & NS & NS & NS \\
\hline $\mathrm{V}_{20}(\%)$ & $\begin{array}{l}96.30 \pm \\
4.45\end{array}$ & $\begin{array}{l}96.08 \\
\pm 4.43\end{array}$ & NS & $\begin{array}{l}96.35 \pm \\
4.24\end{array}$ & $\begin{array}{l}96.01 \pm \\
4.20\end{array}$ & NS & $\begin{array}{l}96.47 \pm \\
4.32\end{array}$ & $\begin{array}{l}96.15 \pm \\
4.25\end{array}$ & NS & NS & NS & NS & NS & 0.008 & NS \\
\hline $\mathrm{D}_{2 \%}(\mathrm{~Gy})$ & $\begin{array}{l}52.88 \pm \\
0.75\end{array}$ & $\begin{array}{l}52.60 \\
\pm 0.73\end{array}$ & NS & $\begin{array}{l}52.79 \pm \\
0.76\end{array}$ & $\begin{array}{l}52.68 \pm \\
0.67\end{array}$ & NS & $\begin{array}{l}52.85 \pm \\
0.75\end{array}$ & $\begin{array}{l}52.70 \pm \\
0.66\end{array}$ & NS & NS & NS & NS & NS & NS & NS \\
\hline$D_{\max }(G y)$ & $\begin{array}{l}54.90 \pm \\
1.29 \\
\end{array}$ & $\begin{array}{l}54.76 \\
\pm 1.17 \\
\end{array}$ & NS & $\begin{array}{l}54.65 \pm \\
1.34\end{array}$ & $\begin{array}{l}54.49 \pm \\
0.73\end{array}$ & NS & $\begin{array}{l}54.52 \pm \\
1.21 \\
\end{array}$ & $\begin{array}{l}54.66 \pm \\
0.89\end{array}$ & NS & 0.042 & NS & 0.022 & NS & NS & NS \\
\hline$D_{\text {mean }}(G y)$ & $\begin{array}{l}52.90 \pm \\
3.28\end{array}$ & $\begin{array}{l}43.49 \\
\pm 2.42 \\
\end{array}$ & NS & $\begin{array}{l}43.14 \pm \\
3.33\end{array}$ & $\begin{array}{l}43.76 \pm \\
2.39\end{array}$ & NS & $\begin{array}{l}43.39 \pm \\
3.24\end{array}$ & $\begin{array}{l}43.87 \pm \\
2.49\end{array}$ & NS & 0.007 & 0.001 & 0 & 0.013 & NS & 0.003 \\
\hline \multicolumn{16}{|l|}{ Bowel } \\
\hline$V_{40}(\%)$ & $\begin{array}{l}4.95 \pm \\
3.41\end{array}$ & $\begin{array}{l}5.28 \pm \\
3.58 \\
\end{array}$ & NS & $4.9 \pm 3.3$ & $\begin{array}{l}5.15 \pm \\
3.66 \\
\end{array}$ & NS & $\begin{array}{l}5.01 \pm \\
3.37\end{array}$ & $\begin{array}{l}5.13 \pm \\
3.62 \\
\end{array}$ & NS & NS & NS & 0.002 & NS & NS & NS \\
\hline $\mathrm{V}_{30}(\%)$ & $\begin{array}{l}23.52 \pm \\
8.71\end{array}$ & $\begin{array}{l}21.52 \\
\pm 9.40\end{array}$ & 0.015 & $\begin{array}{l}22.53 \pm \\
8.66\end{array}$ & $\begin{array}{l}20.87 \pm \\
8.85\end{array}$ & 0.02 & $\begin{array}{l}22.0 \pm \\
8.47\end{array}$ & $\begin{array}{l}20.88 \pm \\
8.97\end{array}$ & NS & 0.002 & NS & 0.002 & 0.02 & 0.01 & 0 \\
\hline $\mathrm{V}_{20}(\%)$ & $\begin{array}{l}50.7 \pm \\
11.99\end{array}$ & $\begin{array}{l}47.98 \\
\pm 8.91\end{array}$ & NS & $\begin{array}{l}50.41 \pm \\
11.93\end{array}$ & $\begin{array}{l}46.85 \pm \\
8.86\end{array}$ & 0.016 & $\begin{array}{l}50.39 \pm \\
12.08\end{array}$ & $\begin{array}{l}46.73 \pm \\
8.85\end{array}$ & 0.015 & 0.017 & NS & NS & 0.024 & NS & 0.024 \\
\hline $\mathrm{V}_{10}(\%)$ & $\begin{array}{l}65.66 \pm \\
12.36\end{array}$ & $\begin{array}{l}62.07 \\
\pm \\
10.81\end{array}$ & 0.002 & $\begin{array}{l}65.85 \pm \\
12.33\end{array}$ & $\begin{array}{l}62.43 \pm \\
10.88\end{array}$ & 0.002 & $\begin{array}{l}66.16 \pm \\
12.23\end{array}$ & $\begin{array}{l}63.14 \pm \\
10.81\end{array}$ & 0.005 & NS & 0 & 0 & 0.001 & 0 & 0 \\
\hline $\mathrm{D}_{\max }(\mathrm{Gy})$ & $\begin{array}{l}52.18 \pm \\
3.57 \\
\end{array}$ & $\begin{array}{l}51.96 \\
\pm 3.30 \\
\end{array}$ & NS & $\begin{array}{l}51.68 \pm \\
3.21 \\
\end{array}$ & $\begin{array}{l}51.58 \pm \\
3.22 \\
\end{array}$ & NS & $\begin{array}{l}51.51 \pm \\
2.99\end{array}$ & $\begin{array}{l}51.74 \pm \\
3.36\end{array}$ & NS & 0.002 & NS & 0.002 & NS & NS & NS \\
\hline $\mathrm{D}_{\text {mean }}(\mathrm{Gy})$ & $\begin{array}{l}18.89 \pm \\
3.82\end{array}$ & $\begin{array}{l}18.43 \\
\pm 3.17 \\
\end{array}$ & NS & $\begin{array}{l}18.63 \pm \\
3.75\end{array}$ & $\begin{array}{l}18.32 \pm \\
3.01\end{array}$ & NS & $\begin{array}{l}18.63 \pm \\
3.77\end{array}$ & $\begin{array}{l}18.12 \pm \\
3.19\end{array}$ & NS & 0 & NS & 0 & NS & NS & 0.001 \\
\hline $\mathrm{V}_{195 \mathrm{cc}}(\mathrm{Gy})$ & $\begin{array}{l}34.85 \pm \\
3.49\end{array}$ & $\begin{array}{l}33.88 \\
\pm 3.77\end{array}$ & 0 & $\begin{array}{l}34.55 \pm \\
3.53\end{array}$ & $\begin{array}{l}33.70 \pm \\
3.74\end{array}$ & 0 & $\begin{array}{l}34.37 \pm \\
3.64\end{array}$ & $\begin{array}{l}33.78 \pm \\
3.83\end{array}$ & 0.003 & 0.004 & NS & NS & 0.035 & 0.047 & 0.003 \\
\hline \multicolumn{16}{|l|}{ RT femur } \\
\hline $\mathrm{V}_{50}(\%)$ & $\begin{array}{l}2.37 \pm \\
2.54\end{array}$ & $0 \pm 0$ & 0.001 & $\begin{array}{l}1.89 \pm \\
2.25\end{array}$ & $0 \pm 0$ & 0.003 & $\begin{array}{l}1.87 \pm \\
2.34\end{array}$ & $0 \pm 0$ & 0.004 & 0.011 & NS & 0.001 & NS & NS & NS \\
\hline $\mathrm{V}_{40}(\%)$ & $\begin{array}{l}4.95 \pm \\
3.41\end{array}$ & $\begin{array}{l}5.27 \pm \\
3.58 \\
\end{array}$ & NS & $4.9 \pm 3.3$ & $\begin{array}{l}5.14 \pm \\
3.66 \\
\end{array}$ & NS & $\begin{array}{l}5.01 \pm \\
3.37 \\
\end{array}$ & $\begin{array}{l}5.12 \pm \\
3.62 \\
\end{array}$ & NS & NS & 0.002 & NS & NS & NS & NS \\
\hline $\mathrm{V}_{30}(\%)$ & $\begin{array}{l}21.52 \pm \\
9.4 \\
\end{array}$ & $\begin{array}{l}20.81 \\
\pm 4.67 \\
\end{array}$ & NS & $\begin{array}{l}20.87 \pm \\
8.87 \\
\end{array}$ & $\begin{array}{l}21.52 \pm \\
3.88 \\
\end{array}$ & NS & $\begin{array}{l}20.88 \pm \\
8.97 \\
\end{array}$ & $\begin{array}{l}21.48 \pm \\
3.91 \\
\end{array}$ & NS & 0.002 & NS & 0.002 & NS & NS & NS \\
\hline$V_{20}(\%)$ & $\begin{array}{l}79.04 \pm \\
17.12 \\
\end{array}$ & $\begin{array}{l}39.47 \\
\pm 5.84 \\
\end{array}$ & 0 & $\begin{array}{l}77.12 \pm \\
17.83\end{array}$ & $\begin{array}{l}39.8 \pm \\
5.17 \\
\end{array}$ & 0 & $\begin{array}{l}76.61 \pm \\
18.01\end{array}$ & $\begin{array}{l}40.57 \pm \\
5.38\end{array}$ & 0 & 0.031 & NS & 0.031 & NS & NS & NS \\
\hline $\mathrm{V}_{10}(\%)$ & $\begin{array}{l}90.97 \pm \\
8.83\end{array}$ & $\begin{array}{l}85.79 \\
\pm \\
11.83\end{array}$ & 0.04 & $\begin{array}{l}91.38 \pm \\
8.53\end{array}$ & $\begin{array}{l}85.42 \pm \\
12.51\end{array}$ & 0.01 & $\begin{array}{l}91.97 \pm \\
8.18\end{array}$ & $\begin{array}{l}83.9 \pm \\
8.65\end{array}$ & 0 & 0.036 & 0.007 & 0.014 & NS & NS & NS \\
\hline$D_{\max }(G y)$ & $\begin{array}{l}53.78 \pm \\
2.66\end{array}$ & $\begin{array}{l}51.99 \\
\pm 2.61 \\
\end{array}$ & 0.001 & $\begin{array}{l}52.95 \pm \\
2.41 \\
\end{array}$ & $\begin{array}{l}51.72 \pm \\
2.34 \\
\end{array}$ & 0.037 & $\begin{array}{l}52.93 \pm \\
2.16 \\
\end{array}$ & $\begin{array}{l}52.13 \pm \\
2.28 \\
\end{array}$ & NS & 0 & NS & 0 & NS & NS & NS \\
\hline$D_{\text {mean }}(G y)$ & $\begin{array}{l}27.34 \pm \\
5.14\end{array}$ & $\begin{array}{l}20.42 \\
\pm 2.03\end{array}$ & 0 & $\begin{array}{l}26.88 \pm \\
5.19\end{array}$ & $\begin{array}{l}20.37 \pm \\
1.74\end{array}$ & 0 & $\begin{array}{l}26.73 \pm \\
5.11\end{array}$ & $\begin{array}{l}20.49 \pm \\
1.85\end{array}$ & 0 & 0.008 & NS & 0.001 & NS & NS & NS \\
\hline \multicolumn{16}{|l|}{ LT femur } \\
\hline $\mathrm{V}_{50}(\%)$ & $\begin{array}{l}2.08 \pm \\
3.04\end{array}$ & $\begin{array}{l}0.68 \pm \\
1.24 \\
\end{array}$ & NS & $\begin{array}{l}1.68 \pm \\
2.64\end{array}$ & $\begin{array}{l}0.73 \pm \\
1.3\end{array}$ & NS & $\begin{array}{l}1.65 \pm \\
2.59\end{array}$ & $\begin{array}{l}0.58 \pm \\
0.94\end{array}$ & NS & 0.018 & NS & 0.004 & NS & NS & NS \\
\hline $\mathrm{V}_{40}(\%)$ & $\begin{array}{l}12.61 \pm \\
8.67\end{array}$ & $\begin{array}{l}8.29 \pm \\
3.28\end{array}$ & 0.032 & $\begin{array}{l}11.75 \pm \\
8.57\end{array}$ & $\begin{array}{l}8.5 \pm \\
3.52\end{array}$ & NS & $\begin{array}{l}11.77 \pm \\
7.79\end{array}$ & $\begin{array}{l}8.54 \pm \\
3.26\end{array}$ & NS & 0.037 & NS & NS & NS & NS & NS \\
\hline $\mathrm{V}_{30}(\%)$ & $\begin{array}{l}38.85 \pm \\
21.9\end{array}$ & $\begin{array}{l}21.02 \\
\pm 4.55 \\
\end{array}$ & 0.004 & $\begin{array}{l}36.57 \pm \\
22.7\end{array}$ & $\begin{array}{l}21.04 \pm \\
4.23\end{array}$ & 0.012 & $\begin{array}{l}36.47 \pm \\
20.24\end{array}$ & $\begin{array}{l}21.22 \pm \\
4.4\end{array}$ & 0.006 & 0.007 & NS & NS & NS & NS & NS \\
\hline $\mathrm{V}_{20}(\%)$ & $\begin{array}{l}77.07 \pm \\
22.31\end{array}$ & $\begin{array}{l}40.6 \pm \\
5.67\end{array}$ & 0 & $\begin{array}{l}73.91 \pm \\
22.94\end{array}$ & $\begin{array}{l}39.53 \pm \\
6.09\end{array}$ & 0 & $\begin{array}{l}77.68 \pm \\
16.71\end{array}$ & $\begin{array}{l}41.02 \pm \\
7.09\end{array}$ & 0 & 0.004 & NS & NS & NS & NS & NS \\
\hline $\mathrm{V}_{10}(\%)$ & $\begin{array}{l}90.30 \pm \\
11.8\end{array}$ & $\begin{array}{l}83.95 \\
\pm \\
11.68 \\
\end{array}$ & 0.022 & $\begin{array}{l}90.35 \pm \\
12.09\end{array}$ & $\begin{array}{l}85.52 \pm \\
12.59\end{array}$ & NS & $\begin{array}{l}92.71 \pm \\
9.49\end{array}$ & $\begin{array}{l}85.5 \pm \\
12.2\end{array}$ & 0.004 & NS & NS & NS & NS & NS & NS \\
\hline$D_{\max }(G y)$ & $\begin{array}{l}52.75 \pm \\
3.25\end{array}$ & $\begin{array}{l}51.50 \\
\pm 2.31\end{array}$ & NS & $\begin{array}{l}52.03 \pm \\
3.04\end{array}$ & $\begin{array}{l}51.45 \pm \\
2.06\end{array}$ & NS & $\begin{array}{l}52.69 \pm \\
1.49\end{array}$ & $\begin{array}{l}51.72 \pm \\
2.22\end{array}$ & 0.046 & 0 & NS & NS & NS & NS & NS \\
\hline
\end{tabular}


Table 2 (continued)

\begin{tabular}{|c|c|c|c|c|c|c|c|c|c|c|c|c|c|c|c|}
\hline \multirow{3}{*}{\begin{tabular}{|l} 
PTV \\
Parameter
\end{tabular}} & \multicolumn{9}{|c|}{ Energy } & \multicolumn{6}{|c|}{$p$-Value } \\
\hline & \multicolumn{3}{|c|}{$6 \mathrm{MV}$} & \multicolumn{3}{|c|}{$10 \mathrm{MV}$} & \multicolumn{3}{|c|}{$15 \mathrm{MV}$} & \multicolumn{3}{|c|}{ IMRT } & \multicolumn{3}{|c|}{ DA } \\
\hline & IMRT & DA & $p$-Value & IMRT & DA & $p$-Value & IMRT & DA & $p$-Value & $\begin{array}{l}6 \text { vs } \\
10\end{array}$ & $\begin{array}{l}10 \text { vs } \\
15\end{array}$ & 6 vs 15 & $\begin{array}{l}6 \text { vs } \\
10\end{array}$ & $\begin{array}{l}10 \text { vs } \\
15\end{array}$ & $\begin{array}{l}6 \text { vs } \\
15\end{array}$ \\
\hline $\mathrm{D}_{\text {mean }}(\mathrm{Gy})$ & $\begin{array}{l}27.03 \pm \\
6.09\end{array}$ & $\begin{array}{l}20.43 \\
\pm 1.79\end{array}$ & 0 & $\begin{array}{l}26.45 \pm \\
6.19\end{array}$ & $\begin{array}{l}20.26 \pm \\
1.92\end{array}$ & $0 t$ & $\begin{array}{l}26.99 \pm \\
4.99\end{array}$ & $\begin{array}{l}20.51 \pm \\
1.99\end{array}$ & 0 & 0.001 & NS & NS & NS & NS & NS \\
\hline \multicolumn{16}{|l|}{ PHY 2.5} \\
\hline$D_{\text {mean }}(G y)$ & $\begin{array}{l}26.94 \pm \\
2.57\end{array}$ & $\begin{array}{l}26.44 \\
\pm 3.97\end{array}$ & NS & $\begin{array}{l}26.43 \pm \\
2.69\end{array}$ & $\begin{array}{l}26.19 \pm \\
3.99\end{array}$ & NS & $\begin{array}{l}26.28 \pm \\
2.71\end{array}$ & $\begin{array}{l}26.04 \pm \\
4.02\end{array}$ & NS & 0 & 0 & 0 & 0 & 0 & 0 \\
\hline \multicolumn{16}{|l|}{ PHY 5.0} \\
\hline $\mathrm{D}_{\text {mean }}(\mathrm{Gy})$ & $\begin{array}{l}16.91 \pm \\
1.38\end{array}$ & $\begin{array}{l}16.46 \\
\pm 1.44\end{array}$ & 0.001 & $\begin{array}{l}16.07 \pm \\
1.33\end{array}$ & $\begin{array}{l}15.89 \pm \\
1.41\end{array}$ & NS & $\begin{array}{l}15.80 \pm \\
1.31\end{array}$ & $\begin{array}{l}15.69 \pm \\
1.41\end{array}$ & NS & 0 & 0 & 0 & 0 & 0 & 0 \\
\hline \multicolumn{16}{|l|}{ TMU } \\
\hline $\begin{array}{l}\text { TMU } \\
\text { Mean }\end{array}$ & $\begin{array}{l}1,646.88 \\
\pm 332.12\end{array}$ & $\begin{array}{l}522.11 \\
\pm \\
33.57\end{array}$ & 0 & $\begin{array}{l}1,463.05 \\
\pm 327.24\end{array}$ & $\begin{array}{l}456.64 \\
\pm 25.31\end{array}$ & 0 & $\begin{array}{l}1,410.11 \\
\pm 305.94\end{array}$ & $\begin{array}{l}430.94 \pm \\
22.63\end{array}$ & 0 & 0 & 0 & 0 & 0 & 0 & 0 \\
\hline
\end{tabular}

Abbreviations: $\mathrm{Cl}$, conformity index; HI, homogeneity index; IMRT, intensity-modulated radiotherapy; DA, dual arc; Dmax, maximum dose; Dmin, minimum dose; Dmean, mean dose; Dmedian, median dose; Dmodal, modal dose; NS, nonsignificant; TMU, total monitor units; PTV, planning target volume.

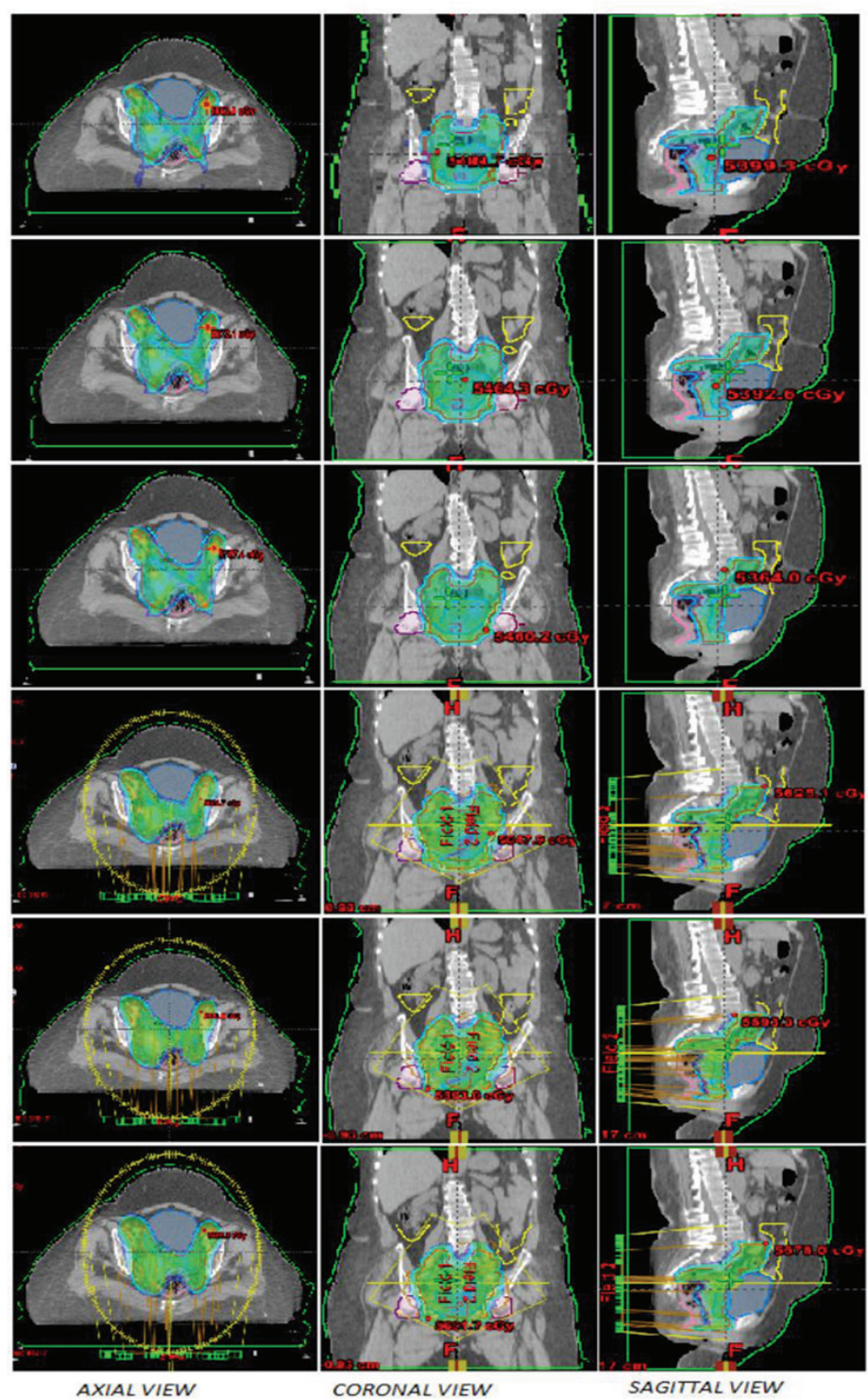

IMRT 6MV

IMRT $10 \mathrm{MV}$

IMRT $15 \mathrm{MV}$

DA $6 \mathrm{MV}$

DA $10 \mathrm{MV}$

DA $15 \mathrm{MV}$

Fig. 2 Dose coverage of low dose regions (28 Gy) for 6 MV, 10 MV, and 15 MV for IMRT and dual arc (DA) plans. IMRT, intensity-modulated radiotherapy. 

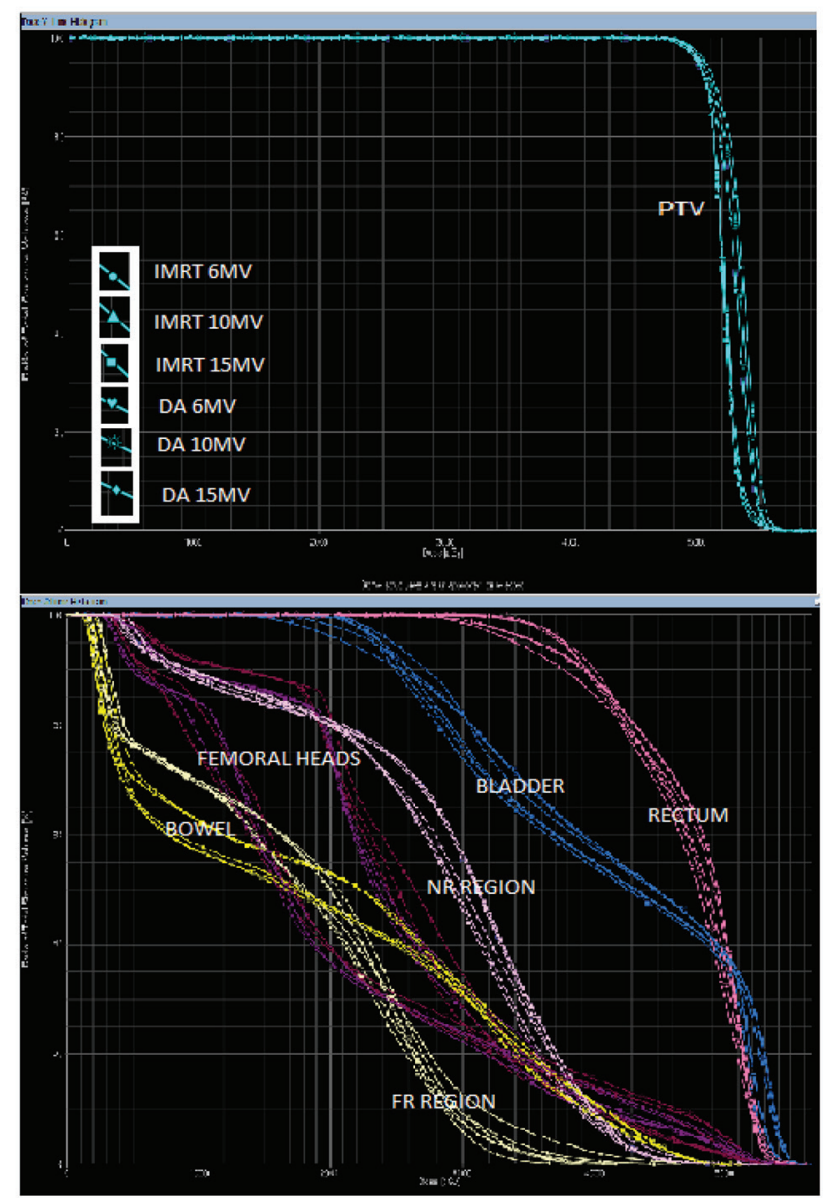

Fig. 3 Cumulative dose volume histogram (DVH) of PTV and OARs. PTV, planning target volume; OARs, organs at risk.

with their respective IMRT plans. The lesser treatment time (irrespective of setup verification time) improves patient comfort, hence leading to improved quality and accuracy of the treatment. The optimum plan aimed at adequate tumor volume coverage with minimal complications should be selected, which is more important in all the cases.

However, the delineation of target volumes, margins, and critical structures varies from center to center and may lead to uncertainty in achieving same dosimetric parameters everywhere. Hence, it is recommended to conduct a multiinstitutional study with a large number of patient populations to validate our observations.

\section{Conclusion}

For treating pelvic or abdominal tumors, DA plan provides a comparable and uncompromised distribution in comparison to intensity-modulated plans with $6 \mathrm{MV}$ photon energy showing more conformal and homogeneous distribution in comparison to $10 \mathrm{MV}$ and $15 \mathrm{MV}$.

For OARs, the lower doses as well as mean doses were lesser for dual arc plans. As the treatment time was reduced for arc plans, the accuracy of the treatment increases significantly. The shorter treatment time and accuracy can lead to better management of cervical cancers.
Considering the production of photo-neutrons during treatment for energies more than $10 \mathrm{MV}$, the DA plans optimized with $6 \mathrm{MV}$ photon energy should be the choice of treatment for carcinoma cervix.

\section{Funding \\ None. \\ Conflict of Interest \\ None declared.}

\section{References}

1 Sankaranarayanan R, Ferlay J. Worldwide burden of gynaecological cancer: the size of the problem. Best Pract Res Clin Obstet Gynaecol 2006;20(2):207-225

2 WHO/ICO Information Centre on HPV and Cervical Cancer (HPV Information Centre), Summary report on HPV and cervical cancer statistics in India 2019. Available at https:// hpvcentre.net/statistics/reports/IND.pdf. Accessed June 17, 2019

3 Intensity Modulated Radiation Therapy Collaborative Working Group. Intensity-modulated radiotherapy: current status and issues of interest. Int J Radiat Oncol Biol Phys 2001;51(4):880-914

4 Adams EJ, Convery DJ, Cosgrove VP, et al. Clinical implementation of dynamic and step-and-shoot IMRT to treat prostate cancer with high risk of pelvic lymph node involvement. Radiother Oncol 2004;70(1):1-10

5 Price RA, Murphy S, McNeeley SW, et al. A method for increased dose conformity and segment reduction for SMLC delivered IMRT treatment of the prostate. Int J Radiat Oncol Biol Phys 2003;57(3):843-852

6 Otto K. Volumetric modulated arc therapy: IMRT in a single gantry arc. Med Phys 2008;35(1):310-317

7 Clivio A, Fogliata A, Franzetti-Pellanda A, et al. Volumetric-modulated arc radiotherapy for carcinomas of the anal canal: A treatment planning comparison with fixed field IMRT. Radiother Oncol 2009;92(1):118-124

8 Söderström S, Eklöf A, Brahme A. Aspects on the optimal photon beam energy for radiation therapy. Acta Oncol 1999;38(2):179-187

9 National Comprehensive Cancer Network (NCCN) recommendations. Available at http://www.nccn.org. Accessed March 10, 2019

10 Solaiappan, et al. Influence of photon beam energy on IMRT plan quality for radiotherapy of prostate cancer. Rep Pract Oncol Radiother 2009;14(1):18-31

11 Laughlin JS, Mohan R, Kutcher GJ. Choice of optimum megavoltage for accelerators for photon beam treatment. Int J Radiat Oncol Biol Phys 1986;12(9):1551-1557

12 William SJ, Mundt AJ, Consensus guidelines for the delineation of the intensity modulated pelvic radiotherapy CTV in the postoperative treatment of endometrial and cervical cancer. Available at https://www.rtog.org/CoreLab/ContouringAtlases/GYN.aspx. Accessed March 10, 2019

13 Isabelle Gagne S, Zavgorodni. Evaluation of the analytical anisotropic algorithm (AAA) in an extreme water-lung interface phantom using Monte Carlo dose calculations. J Appl Clin Med Phys 2007;8(1):33:46

14 Vanetti E, Nicolini G, Nord J, et al. On the role of the optimization algorithm of RapidArc( $\left({ }^{\circledR}\right)$ volumetric modulated arc therapy on plan quality and efficiency. Med Phys 2011;38(11):5844-5856

15 Prescribing, recording and reporting photon beam therapy. (supplement to ICRU report 50) ICRU REPORT 62. ICRU, PP. IX+52, 1999 (ICRU BETHESDA, MD) 
16 Zhai DY, Yin Y, Gong GZ, et al. RapidArc radiotherapy for whole pelvic lymph node in cervical cancer with 6 and $15 \mathrm{MV}$ : a treatment planning comparison with fixed field IMRT. J Radiat Res (Tokyo) 2013;54(1):166-173

17 Pirzkall A, Carol MP, Pickett B, Xia P, Roach M, III. Verhey LJ. The effect of beam energy and number of fields on photon-based IMRT for deep-seated targets. Int J Radiat Oncol Biol Phys 2002;53(2):434-442

18 Garrison H, Anderson J, Laughlin JS, Harvey RA. Comparison of dose distributions in patients treated with $\mathrm{x}$-ray beams of widely different energies. Radiology 1952;58(3):361-368

19 Kry SF, Followill D, White RA, Stovall M, Kuban DA, Salehpour M. Uncertainty of calculated risk estimates for secondary malignancies after radiotherapy. Int J Radiat Oncol Biol Phys 2007;68(4):1265-1271

20 Kry SF, Salehpour M, Followill DS, et al. The calculated risk of fatal secondary malignancies from intensity-modulated radiation therapy. Int J Radiat Oncol Biol Phys 2005;62(4):1195-1203

21 Verbakel WF, Cuijpers JP, Hoffmans D, Bieker M, Slotman BJ, Senan S. Volumetric intensity-modulated arc therapy vs. conventional IMRT in head-and-neck cancer: a comparative planning and dosimetric study. Int J Radiat Oncol Biol Phys 2009;74(1):252-259

22 Doornaert P, Verbakel WF, Bieker M, Slotman BJ, Senan S. RapidArc planning and delivery in patients with locally advanced head-and-neck cancer undergoing chemoradiotherapy. Int J Radiat Oncol Biol Phys 2011;79(2):429-435

23 Cozzi L, Dinshaw KA, Shrivastava SK, et al. A treatment planning study comparing volumetric arc modulation with RapidArc and fixed field IMRT for cervix uteri radiotherapy. Radiother Oncol 2008;89(2):180-191
24 Lui et al. Dosimetric comparison between rapid arc and conventional fixed-field intensity-modulated radiotherapy for prostate cancer. Biomed Imaging Interv J 2013;3:e17

25 Ost P, Speleers B, De Meerleer G, et al. Volumetric arc therapy and intensity-modulated radiotherapy for primary prostate radiotherapy with simultaneous integrated boost to intraprostatic lesion with 6 and $18 \mathrm{MV}$ : a planning comparison study. Int J Radiat Oncol Biol Phys 2011;79(3):920-926

26 Blomquist M, Li J, Ma CM, Zackrisson B, Karlsson M. Comparison between a conventional treatment energy and $50 \mathrm{MV}$ photons for the treatment of lung tumours. Phys Med Biol 2002;47(6):889-897

27 Liu HH, Wang X, Dong L, et al. Feasibility of sparing lung and other thoracic structures with intensity-modulated radiotherapy for non-small-cell lung cancer. Int J Radiat Oncol Biol Phys 2004;58(4):1268-1279

28 Tyagi A, Supe SS, Sandeep , Singh MP. A dosimetric analysis of $6 \mathrm{MV}$ versus $15 \mathrm{MV}$ photon energy plans for intensity modulated radiation therapy (IMRT) of carcinoma of cervix. Rep Pract Oncol Radiother 2010;15(5):125-131

29 Yadav G, Bhushan M, Dewan A, et al. Dosimetric influence of photon beam energy and number of arcs on volumetric modulated arc therapy in carcinoma cervix: A planning study. Rep Pract Oncol Radiother 2017;22(1):1-9

30 Bhushan M, Yadav G, Tripathi D, et al. Dosimetric analysis of unflattened (FFFB) and flattened (FB) photon beam energy for gastric cancers using IMRT and VMAT- a comparative study. J Gastrointest Canc 2018 doi: 10.1007/s12029-018-0080-9

Erratum: Figures 2 and 3 have been corrected as per Erratum published online on August 09, 2019. DOI of the Erratum is $10.1055 / \mathrm{s}-0039-1696062$. 